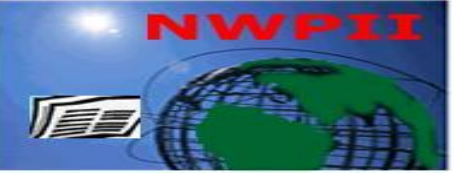

American Journal of Biomedical Sciences

ISSN: 1937-9080

nwpii.com/ajbms

\title{
Fast Computational Analysis of Sway Area Using Center of Pressure Data in Normal Children and Children with Cerebral Palsy
}

\author{
GyuTae Kim $^{1}$, Mohammed Ferdjallah ${ }^{2}$, Gerald F. Harris ${ }^{3}$ \\ ${ }^{1}$ Department of Electrical \& Computer Engineering, Old Dominion University, Norfolk, USA \\ ${ }^{2}$ Virginia Modeling, Analysis \& Simulation Center, Old Dominion University, Norfolk, USA \\ ${ }^{3}$ Orthopaedic \& Rehabilitation Engineering Center, Marquette University, Milwaukee, USA \\ *Corresponding Author: \\ Dr. Mohammed Ferdjallah \\ Virginia Modeling, Analysis and Simulation Center \\ Old Dominion University \\ 1030 University Blvd., Suffolk, VA. 23435, USA \\ Tel: 757-638-4440; Fax: 757-686-6214
}

Received: March 31, 2009; | Revised: 21 June 2009; | Accepted: 2 July 2009

\begin{abstract}
In this paper, we have developed an efficient method for real time computation of Center of Pressure $(C o P)$ sway area using a fast search and sort based on a numerical approximation of the Green-Gauss method. In addition, we investigated the correlations among the velocity, acceleration, path length, sway area, and mean frequency of $C o P$ signals during quiet standing in healthy children and in children with cerebral palsy. The Pearson correlation technique described the direction and strength of linear relationships among the $C o P$ velocity, acceleration, and the standard metrics, such as $C o P$ Root Mean Squared $(R M S)$, CoP range, path length $(P L)$, normalized path length $(N P L)$, sway area $(S A)$, normalized sway area $(N S A)$, and mean frequency, respectively. Subjects were tested during eyes open and eyes closed trials to explore the significance of the visual input. Both $R M S$ of $C o P$ velocity and acceleration described strong correlations with NPL and NSA in the eye open test $(\mathrm{r} \geq 0.728)$ and in the eye closed test $(\mathrm{r} \geq 0.919)$ of normal children. Similar results were also observed in children with cerebral palsy in both eye tests. The correlation between the $C o P$ velocity and NPL indicated that the $C o P$ velocity metric can be considered as an indicator of NPL. Thus, the CoP velocity and $C o P$ acceleration might be very useful as valid metrics because the real time feedback from online measurements in balance control assessment is often necessary. The described fast computation of the sway area by using $C o P$ velocity and $C o P$ acceleration provided valid metrics for $\mathrm{CoP}$ correlation analysis.
\end{abstract}

Keywords: apoptosis Center of Pressure; Center of Pressure Velocity; Center of Pressure Acceleration; Path length; Sway area 


\section{INTRODUCTION}

The ability to perform routine activities of daily life requires the stable control of posture and balance. Stable control of balance is the result of coordinated attempts by the nervous system to maintain balance by integrating proprioceptive, vestibular, visual, motor, and biomechanical information. For vestibularly impaired patients, children with cerebral palsy, and others lacking balance stability due to pathology or injury, maintaining balance is a challenging task. Children with cerebral palsy frequently have an impaired sense of equilibrium, abnormal motor control, persistence of primitive reflexes, and abnormal posturing [1]. Traumatic head and spinal cord injuries often lead to postural stability dysfunctions. In the young, normal physical coordination is an evolutionary process with increased steadiness during each passing year. Children with muscular dystrophy $(M D)$ show diminishing ambulatory skills until wheelchair is needed. Unlike $M D$, cerebral palsy stabilizes usually by age 12 . The elderly are a large section of society that can benefit greatly by improved clinical use of postural control studies. Rehabilitation and therapeutic innervations, which are intended to improve balance control, should lead to improvements in function. Clinical assessment of balance control is therefore critical and often requires integrated functional, systems and quantitative approaches [2,3].

Cerebral palsy is a non-progressive movement disorder often caused by damage to the motor cortex. In most cases, the damage to the motor cortex occurs while giving birth or before birth. Thus, cerebral palsy affects body conditions of an affected child from birth. Although cerebral palsy is an incurable condition, an early diagnosis can provide children's better living condition by helping them adapt to their challenging circumstances. In addition, specialized medical devices for children with cerebral palsy are required for proper medical care. Muscle abnormalities and bone deformities can be partially corrected by medical devices and assistive technologies. Movement analysis and postural stability assessment play a major role in the treatment and prevention of complications arising from cerebral palsy. In particular, assessment of balance control through online measures can be used to design effective orthotics to improve standing balance in children with cerebral palsy.

Stable balance is defined as the ability to maintain and control the body center of mass within the base of support to prevent falls and to complete desired movements. The control of postural stability during quiet standing involves three basic coordination patterns: ankle strategy, hip strategy, and stepping strategy, which have been described in both adults and children [4]. These coordination patterns are developed through acquired learning and fully exhibited between the ages of 7 and 10 years. Because of the complexity of the musculoskeletal system, motor coordination during any strategy has a larger number of options of muscle activation. Consequently, children with cerebral palsy may favor one strategy over another or may adopt a unique biomechanical postural alignment to compensate for muscular weakness and motor deficits $[5,6]$.

The assessment of posture has been extensively studied by measuring center of mass $(C o M)$ and center of pressure $(C o P)$ [7]. The $C o P$ is an indirect measure of postural sway and it is often used instead of CoM for simplicity of instrumentation [8]. The estimation of $C o M$, on the other hand, requires inverse dynamic analysis of a computerized multi-joint model driven by kinematic measurements using an integrated motion analysis system [9]. Accurate estimation of small movements of body $\mathrm{CoM}$ requires very precise measures of proximal and distal displacements of all individual body segments. The $C o P$ is defined as the projection of the center of gravity into the ground and is an indirect measure of body sway. The $C o P$ is a jointly described by $A-P$ (Anterior-Posterior) and $M-L$ (Medial-Lateral) coordinates. The $C O P$ in both anterior-posterior $(A-P)$ and medial-lateral $(M-L)$ planes has proven to be a significant and reliable output metric [10]. The path length, sway area and $A-P$ sway ranges have also been shown to be effective parameters for monitoring postural sway.

Sway in stance is not a direct measure of functional postural stability. If a subject sways slowly as an inverted pendulum, movement of 
CoP forces at the surface is approximately correlated with the position of the body's CoM [11]. When sway is rapid or when the knees and the hips move, the excursion of $\mathrm{CoP}$ reflects kinematics and dynamics of body motion and not just the position of CoM. Therefore, the CoP could be used as an inexpensive alternative to estimate the movement of the center of gravity of the subject to give a more accurate representation of the brain's ability to correct balance. The purpose of this study was to investigate the correlations between the sway velocity, the sway acceleration, the path length, sway area, and the sway mean frequency during quiet standing in healthy children and in children with cerebral palsy. Using dual force platforms, we designed fast search methods to estimate an optimum close and continuous curve, which encompasses the zigzagged trajectory of the $C o P$ signals. The sway area was then computed using a numerical approximation of the Gauss-Green formula. All subjects were tested during eyes open and eyes closed trials to investigate the significance of visual input on these control metrics.

\section{METHODOLOGY}

\subsection{Center of Pressure Analysis}

A single force platform measures the net body $\mathrm{CoP}\left(\mathrm{CoP}_{n e t}\right)$ and its coordinates $\left(x_{\text {net }}, y_{\text {net }}\right)$. When using dual force platforms, the net coordinates can be calculated from $\mathrm{CoPs}$ measured under each limb. The right and left force platforms measure respectively the coordinates $\left(x_{\text {right }}, y_{\text {right }}\right)$ of $\mathrm{CoP}_{\text {right }}$ and the coordinates $\left(x_{\text {left }}, y_{\text {left }}\right)$ of $\mathrm{CoP}_{\text {left }}$. Each platform simultaneously measures three force components along the XYZ axes and three moment components about the XYZ axes. The net coordinates of the $C o P$ can be calculated as follows [13]:

$$
\begin{aligned}
& x_{\text {net }}=\frac{R_{\text {right }}}{R_{\text {right }}+R_{\text {left }}} x_{\text {right }}+\frac{R_{\text {left }}}{R_{\text {right }}+R_{\text {left }}} x_{\text {left }} \\
& y_{\text {net }}=\frac{R_{\text {right }}}{R_{\text {right }}+R_{\text {left }}} y_{\text {right }}+\frac{R_{\text {left }}}{R_{\text {right }}+R_{\text {left }}} y_{\text {left }}
\end{aligned}
$$

where $R_{\text {right }}$ and $R_{\text {left }}$ are the magnitudes of vertical ground reaction forces under the right and left limbs respectively. The coordinate $x_{n e t}$ represents sway in the $A-P$ plan, and the coordinate $y_{n e t}$ represents sway in the $M-L$ plane. The $C o P$ signal reflects the complex neuromuscular feedback network involved in maintaining standing balance. Standing balance control is a biomechanical system, which has energy dissipation mechanism to damp out unwanted oscillatory motions [13]. The fast varying signal in the $C o P$ has been shown to be proportional to the horizontal acceleration of the $C o M$ in $A-P$ and $M-L$ planes. In this study we computed the $C o P$ velocity and acceleration as means to reduce the effects of the slowly varying signal in the $C o P$. Different data were used as a simple approach to remove trends in the CoP time series data and to identify the fast varying component. In this way, we attempted to provide more reliable metrics, which had less dependence of the initial conditions of sway.

The sway pattern of a subject depends on many factors such as initial conditions (initial velocity, weight, and height), which in general are not controlled. Therefore, the sway root mean squared $(R M S)$ and sway range values are also dependent on these initial conditions. Although $R M S$ and range values are still used in balance control, many authors have critical concerns in using these metrics in clinical outcomes. The discrete sway $R M S$ values were calculated as follows:

$$
\begin{aligned}
& x_{R M S}=\frac{1}{N} \sqrt{\sum_{n=1}^{N}\left(x_{n e t}(n)-\bar{x}_{n e t}\right)^{2}} \\
& y_{R M S}=\frac{1}{N} \sqrt{\sum_{n=1}^{N}\left(y_{n e t}(n)-\bar{y}_{n e t}\right)^{2}}
\end{aligned}
$$

where $N$ is the number of data points. The $R M S$ values are biased estimators of the excess energy over the total mean energies of the $C o P$ signals. Because the range values may include unexpected extreme $\mathrm{CoP}$ excursions, they may not reflect the overall characteristics of the sway. Therefore, $\mathrm{CoP}$ data was screened prior to analysis, a task, which may not be accurately performed in automated data processing.

CoP path length measures the distance covered by the center of pressure as it oscillates 
within the base of support. The path length $(P L)$ is evaluated using the following expressions:

$$
P L=\sum_{n=1}^{N} \sqrt{\left[x_{n e t}(n)-x_{n e t}(n-1)\right]^{2}+\left[y_{n e t}(n)-y_{n e t}(n-1)\right]^{2}}
$$

The path length $(P L)$ depends on certain initial conditions more than others. The expression of path length describes the dependency of the results on the absolute difference between successive CoP positions rather than their individual magnitudes. Therefore, the initial conditions and subject's weight and height affect the path length less than the $R M S$ and range values.

\subsection{Sway Area}

The sway area describes the enclosed area covered by the $C o P$ as it oscillates within the base of support. The curve representing by $\left(x_{n e t}, y_{\text {net }}\right)$ may overlap several times. Consequently, calculating the sway area using the Gauss-Green theorem is impossible. Therefore, a general method in the computation of sway area divides the rectangular area into two-dimensional grid meshes. The area is defined by the differences between the maximum and minimum in $x_{\text {net }}$ and $y_{\text {net }}$. A search is then performed to determine all grid points, which are enclosed by the $C o P$ trajectory. The grid points are then summed to form the sway area. The resolution of the mesh defines the accuracy of the area measured. A smaller grid mesh would result in accurate measurement. However, the search protocol would take longer to implement. A larger grid mesh would result in a faster search with less accurate measurement of area.

The Principal Component Analysis (PCA) is another method to calculate sway area $[16,17]$. In $P C A, C o P$ data points are expressed as polar coordinates [17]. Next, the furthest points from the center are found. These points form the first approximation for sway area [17]. However, the $P C A$ method considers the sway area as a roughly rounded shape similar to an ellipse. Thus, the resultant calculated sway area covers the densest region, and often fails to detect data outside the first approximation area. The PCA method gives acceptable results, but the process is very complicated and time consuming. Therefore, a simple and fast computation for sway area is required. The proposed method below can deliver a fast and accurate computation for sway area.

The fast algorithm consists of the following steps:

1. Define a two-dimensional grid with size $N$ equal to the number of $C o P$ data points. The resolution of the gird is estimated by dividing the path length by $N$.

2. Find the grid points, which cross the points $\left(x_{\text {net }}, y_{\text {net }}\right)$ of the data set.

3. Interpolate and find the grid points, which cross the lines connecting the points $\left[x_{\text {net }}(n+1), y_{\text {net }}(n+1)\right]$ and $\left[x_{\text {net }}(n), y_{\text {net }}(n)\right]$.

4. Compute a first closed and continuous contour by searching the most extreme gird points. The $x$-coordinates of the outer grid points are found by incrementing the $y$ coordinates using the resolution of the grid established in the first step. If two adjacent grid points have the same $x$-coordinate, one is eliminated. If more than two adjacent grid points have the same $x$-coordinate, only the extreme grid points are kept.

5. Compute a second contour by searching the most extreme grid points by incrementing through the $x$-axis. The adjacent grid points, which have the same $y$-coordinate, should be purged in the same manner as in the previous step.

6. Align the two contours and merge the sets of grid data points to form an optimum closed and counterclockwise curve.

7. Finally, apply the Gauss-Green theorem to find the area enclosed by the estimated contour.

The final contour, defined by the grid points $\{x, y\}$, is a closed parameterization of the region, which enclose the $C o P$ zigzagged trajectory. The area enclosed can then be estimated using the Gauss-Green formula given by:

$S A=\frac{1}{2} \int\left[x(t) y^{\prime}(t)-x^{\prime}(t) y(t)\right] d t$

A numerical approximation of the Gauss-Green formula can be derived as follows: 
$S A=\frac{1}{4} \sum_{i=1}^{M}\left[\left(x_{i+1}+x_{i}\right)\left(y_{i+1}-y_{i}\right)-\left(x_{i+1}-x_{i}\right)\left(y_{i+1}+y_{i}\right)\right]$

where $M$ is the number of grid points in the optimum contour. Because subjects may stand on the force platforms for slightly different lengths of time, the sway area is generally normalized to the time interval of standing to yield the normalized sway area (NSA). Consequently, the normalized path length $(N P L)$ has the unit of velocity.

\subsection{CoP Mean Frequency}

The analysis of $\mathrm{CoP}$ signals in the frequency domain seeks to measure parameters, which describe specific aspects of the frequency spectrum of the signal. The spectral analysis of CoP data determines if there were any changes in selected spectral parameters between healthy children and children with cerebral palsy. In particular, the mean frequency, which is defined as the centroid of the spectrum, is a useful tool [15]. The mean frequency reflects a global shift in the power distribution. To calculate the mean frequency, the following expression was used:

$$
f_{\text {mean }}=\frac{1}{\pi} \frac{\int \omega\left\|F(\omega) F^{*}(\omega)\right\| d \omega}{\int\left\|F(\omega) F^{*}(\omega)\right\| d \omega}
$$

where $F(\omega)$ is the Fourier transform of the CoP data respectively in the $A-P$ and $M-L$ planes. The Fast Fourier Transform (FFT) is used to estimate the frequency distribution of $\mathrm{CoP}$ signals. The mean frequency has proven to be a clinical marker in balance control assessment.

\subsection{Subjects}

Eight healthy control children (2 girls and 6 boys) aged between 5 and 13 years $\{$ age $=8.8$ years (SD 2.7); weight $=28.2 \mathrm{~kg}$ (SD 12.0); height $=128$ $\mathrm{cm}$ (SD 19)\}, and fifty four independent ambulatory children with diplegic cerebral palsy (19 girls and 35 boys) aged between 6 and 16 years $\{$ age $=9.9$ years $(\mathrm{SD} 3.5)$; weight $=29.6 \mathrm{~kg}$ (SD 11.0); height $=130 \mathrm{~cm}$ (SD 15) $\}$ participated in this study. The healthy controls were screened by clinical survey to have no history of head injury, neurological, vestibular or musculoskeletal dysfunction, or gross abnormalities. All children were screened by an orthopaedic physician prior to participation in the study. During standing, children with cerebral palsy were able to place their feet flat on the force platforms. Informed consent was obtained from the children's guardians prior to participation in the study.

\subsection{Protocol and Data Analysis}

The center of pressure signals were collected at a sampling frequency of $20 \mathrm{~Hz}$ using two standard force platforms (AMTI OR6-5-1, Advanced Mechanical Technology, Newton, MA, USA) interfaced to a PC/Pentium $800 \mathrm{MHz}$. The subjects were instructed to maintain a quiet comfortable stance for 20 seconds while barefoot with each foot on one force platform, heels aligned and arms comfortably on the sides. The subjects were free to select the angulation and medial/lateral spacing of their feet. CoP data was then recorded for 20 to 25 seconds. The duration of 20 to 25 seconds was selected to ensure the stationarity of $C o P$ signals. Recent studies have shown that the CoP may be non-stationary when recorded for longer durations, typically exceeding 30 seconds $[16,17,18,19]$. Data were collected in two consecutive trials where the subjects were asked either to open or close their eyes throughout the stance period. A seated period of 120 seconds was provided for patient relaxation after each test. Prior to analysis, the center of pressure components $(x, y)$, were calculated from force and moment data measured by each force platform. DC offsets were subsequently removed. Only relative changes in $C o P$ movements were considered in this study. These signals were passed through a Hanning window, and filtered (with a dual pass to remove phase shift) using a fourth-order Butterworth digital lowpass filter with a cutoff frequency of $5.0 \mathrm{~Hz}$. The $C o P$ data was then processed to compute the RMS values, range values, velocity, acceleration, path length, area, and mean frequency for $A-P$ and $M-L$ sways.

\section{RESULT}

Figure 1 shows a sample of $C o P$ data in the $A-P$ plane of a typical healthy child during eyes open $(E O)$ test. The $C o P$ velocity and 
acceleration are also illustrated in the same figure for visual comparison.

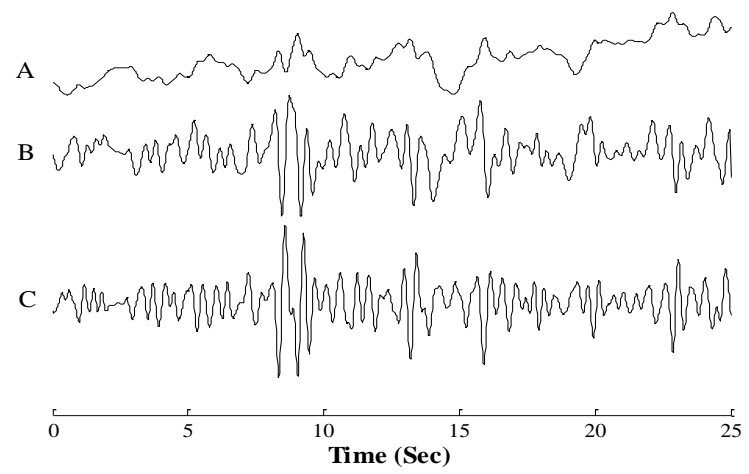

Fig. 1: Time domain samples of 25 seconds of $\mathrm{CoP}$ time series (A), $C o P$ velocity (B), and $C o P$ acceleration $(\mathrm{C})$ in the $A-P$ plan for a healthy child during eyes open test in quiet standing

The sample signal $x_{n e t}$ exhibits an oscillatory behavior composed of a slowly varying signal and a fast varying signal. The slowly varying signal is reduced in the $C o P$ velocity $v x_{n e t}$ and $C o P$ acceleration $a x_{n e t}$. Figure 2 illustrates a closed contour sample, for the same normal child, calculated by the fast method developed in this study. The sway area was calculated by applying the Gauss-Green formula as described in the methods section. Table 1 summarizes the mean and standard deviation values of $R M S$, ranges, path length, normalized path length, sway area, normalized sway area, $R M S$ velocities, $R M S$ accelerations, and mean frequencies of $C o P$ components for healthy children and children with cerebral palsy. The table lists these values during eyes open $(E O)$ and eyes closed $(E C)$ tests. The T-test (paired, two tailed, $p<0.05$ ) was applied to determine the statistical significance between the eyes open and the eyes closed tests. In healthy children, the path length $(P L)$ and the normalized path length $(N P L)$ were significantly $(p<0.01)$ higher during the eyes closed test when compared to the eyes open test. The sway area $(S A)$ and the normalized sway area (NSA) increased during the eyes closed test. Similarly, the $R M S$ of A-P CoP velocity $\left(v x_{R M S}\right), R M S$ of $A-P C O P$ acceleration $\left(a x_{R M S}\right)$, and mean frequencies $\left(f x_{\text {mean }}, f y_{\text {mean }}\right)$ also significantly $(p<0.001)$ increased in the eyes closed test. The $R M S$ of $M-L C O P$ velocity $\left(v y_{R M S}\right)$ and $R M S$ of $M-L C O P$ acceleration $\left(a y_{R M S}\right)$ increased during eyes closed test but not significantly $(p>0.1)$. However, the CoP RMS $\left(x_{R M S}, y_{R M S}\right)$ and ranges ( $\left.x_{\text {range }}, y_{\text {range }}\right)$ decreased during the eyes closed test.

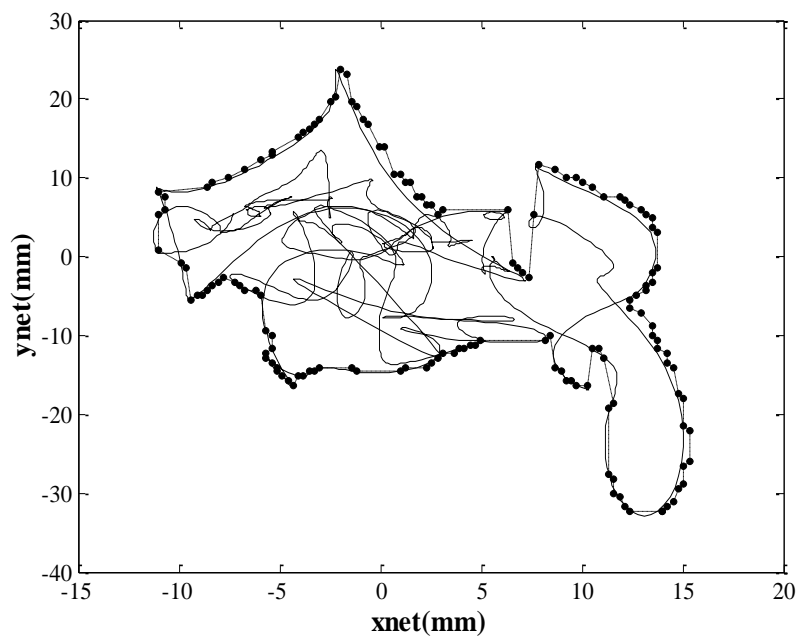

Fig. 2: Illustration samples of the trajectory of $C o P_{n e t}$ (solid line) and the enclosed contour computed by the fast method designed in this study (dotted line) for a healthy child during eyes open test in quiet standing

In the children with cerebral palsy, the path length $(P L)$ and the normalized path length $(N P L)$ also significantly $(p<0.02)$ increased during the eyes closed test. However, the sway area $(S A)$ and the normalized sway (NSA) decreased during the eyes closed test. The path length $(P L)$, the normalized path length $(N P L)$, the sway area $(S A)$, and the normalized sway area (NSA) are significantly $(p<0.005)$ less than those of healthy children in both the eyes open and eyes closed tests. The RMS of A-P CoP velocity $\left(v x_{R M S}\right)$ and the $R M S$ of $A-P$ acceleration $\left(a x_{R M S}\right)$ increased significantly $(p<0.03)$ during the eyes closed test, while the $R M S$ of $M-L C o P$ velocity $\left(v y_{R M S}\right)$ and the $R M S$ of $M-L$ acceleration $\left(a y_{R M S}\right)$ remained relatively constant. The mean frequencies $\left(f x_{\text {mean }}\right.$, $\left.f y_{\text {mean }}\right)$ significantly $(p<0.04)$ increased during the eyes closed test. No consistent patterns were observed in the CoP RMS ( $\left.x_{R M S}, y_{R M S}\right)$ and ranges values ( $\left.x_{\text {range }}, y_{\text {range }}\right)$.

To assess the relationships among the $\mathrm{CoP}$ velocity, $C o P$ acceleration, path length, area, and mean frequency the Pearson product moment 
correlation $(r)$ was used. Table 2 summarizes the $r$ values between the $C o P$ velocity and $C o P$ acceleration respectively paired with $C o P R M S$ $\left(x_{R M S}, y_{R M S}\right), C o P$ ranges $\left(x_{\text {range }}, y_{\text {range }}\right)$, normalized path length $(N P L)$, normalized sway area (NSA), and mean frequencies $\left(f x_{\text {mean }}, f y_{\text {mean }}\right)$ for healthy children and children with cerebral palsy during eyes open and eyes closed tests. In healthy children, both the RMS of $C o P$ velocity and $C o P$ acceleration were correlated to $\mathrm{CoP} R M S, C o P$ ranges, normalized path length, and normalized sway area $(r \geq 0.667)$ in both the $A-P$ and $M-L$ planes during eyes open and eyes closed tests. In particular, there were strong correlations between the $R M S$ of $C o P$ velocity $\left(v x_{R M S}, v y_{R M S}\right)$ and $C o P$ acceleration $\left(a x_{R M S}, a y_{R M S}\right)$ for normalized path length $(N P L)$ and normalized sway area (NSA) respectively. The mean frequency in the $A-P$ plane $\left(f x_{\text {mean }}\right)$ showed mild correlation to the $R M S$ of $C o P$ velocity $\left(v x_{R M S}\right)$ and $C o P$ acceleration $\left(a x_{R M S}\right)$. Poor correlation was observed in the $M-L$ plane between the mean frequency $\left(f y_{\text {mean }}\right)$ and the $R M S$ of $C o P$ velocity and $C o P$ acceleration ( $v y_{R M S}$, $\left.a y_{R M S}\right)$.

Table 1: Mean and standard deviation values of $C o P$ $R M S$, rnage, velocity, acceleration, path length $(P L)$, normalized path length (NPL), sway area $(S A)$, normalized sway area (NSA), and mean frequency in $A$ $P$ and $M-L$ planes for healthy children and children with cerebral palsy during eyes open an eyes closed tests in quiet standing

\begin{tabular}{|c|c|c|c|c|}
\hline & \multicolumn{2}{|c|}{ Normal Children $(n=8)$} & \multicolumn{2}{|c|}{ Children with CP $(n=54)$} \\
\hline & $E O$ & $E C$ & $E O$ & $E C$ \\
\hline$x_{R M S}(\mathrm{~cm})$ & $0.9 \pm 0.5$ & $0.8 \pm 0.3$ & $1.0 \pm 0.4$ & $1.0 \pm 0.5$ \\
\hline$y_{R M S}(\mathrm{~cm})$ & $1.1 \pm 0.8$ & $1.0 \pm 0.6$ & $1.1 \pm 1.0$ & $0.8 \pm 0.3$ \\
\hline$x_{\text {range }}(\mathrm{cm})$ & $4.3 \pm 1.8$ & $4.3 \pm 1.7$ & $4.5 \pm 2.0$ & $4.8 \pm 1.8$ \\
\hline$y_{\text {range }}(\mathrm{cm})$ & $6.5 \pm 3.9$ & $5.8 \pm 3.1$ & $5.3 \pm 5.2$ & $3.7 \pm 1.3$ \\
\hline$P L(\mathrm{~cm})$ & $79.0 \pm 37.1$ & $92.2 \pm 52.8$ & $53.2 \pm 33.4$ & $65.5 \pm 22.5$ \\
\hline $\begin{array}{c}N P L \\
(\mathrm{~cm} / \mathrm{sec})\end{array}$ & $3.2 \pm 1.5$ & $3.7 \pm 2.1$ & $2.1 \pm 1.3$ & $2.6 \pm 0.9$ \\
\hline$S A\left(\mathrm{~cm}^{2}\right)$ & $15.0 \pm 17.3$ & $15.9 \pm 16.1$ & $9.5 \pm 8.8$ & $7.1 \pm 4.5$ \\
\hline $\begin{array}{c}N S A \\
\left(\mathrm{~cm}^{2} / \mathrm{sec}\right)\end{array}$ & $0.6 \pm 0.7$ & $0.6 \pm 0.6$ & $0.4 \pm 0.4$ & $0.3 \pm 0.2$ \\
\hline $\begin{array}{c}v x_{R M S} \\
(\mathrm{~cm} / \mathrm{sec})\end{array}$ & $2.3 \pm 1.4$ & $2.9 \pm 1.8$ & $1.6 \pm 0.7$ & $1.9 \pm 0.8$ \\
\hline $\begin{array}{c}v y_{R M S} \\
(\mathrm{~cm} / \mathrm{sec})\end{array}$ & $3.3 \pm 1.4$ & $3.5 \pm 1.9$ & $2.0 \pm 1.8$ & $2.0 \pm 0.9$ \\
\hline $\begin{array}{c}a x_{R M S} \\
\left(\mathrm{~cm} / \mathrm{sec}^{2}\right)\end{array}$ & $18.6 \pm 10.1$ & $27.1 \pm 19.8$ & $12.2 \pm 6.1$ & $16.1 \pm 6.9$ \\
\hline $\begin{array}{c}a y_{R M S} \\
\left(\mathrm{~cm} / \mathrm{sec}^{2}\right)\end{array}$ & $28.8 \pm 9.6$ & $31.6 \pm 16.6$ & $14.5 \pm 10.4$ & $14.0 \pm 8.0$ \\
\hline$f x_{\text {mean }}(\mathrm{Hz})$ & $0.3 \pm 0.1$ & $0.4 \pm 0.2$ & $0.2 \pm 0.1$ & $0.4 \pm 0.1$ \\
\hline$f y_{\text {mean }}(\mathrm{Hz})$ & $0.4 \pm 0.1$ & $0.4 \pm 0.1$ & $0.3 \pm 0.1$ & $0.3 \pm 0.1$ \\
\hline
\end{tabular}

Am. J. Biomed. Sci. 2009, 1(4), 364-372; doi: 10.5099/aj090400364
Table 2: Pearson correlation factors between the $R M S$ of $C o P$ velocity and $C o P$ acceleration and the other metrics such as CoP RMS, CoP range, path length $(P L)$, normalized path length $(N P L)$, sway area $(S A)$, normalized sway area (NSA), and mean frequency respectively in $A-P$ and $M-L$ planes for healthy children and children with cerebral palsy during eyes open an eyes closed tests in quiet standing

\begin{tabular}{|c|c|c|c|c|c|c|c|c|}
\hline \multirow{3}{*}{$A-P$} & \multicolumn{4}{|c|}{ Normal Children $(n=8)$} & \multicolumn{4}{|c|}{ Children with CP (n=54) } \\
\hline & \multicolumn{2}{|c|}{$v x_{R M S}$} & \multicolumn{2}{|c|}{$a x_{R M S}$} & \multicolumn{2}{|c|}{$v x_{R M S}$} & \multicolumn{2}{|c|}{$a x_{R M S}$} \\
\hline & $E O$ & $E C$ & $E O$ & $E C$ & $E O$ & $E C$ & $E O$ & $E C$ \\
\hline$x_{R M S}$ & 0.66 & 0.78 & 0.65 & 0.65 & 0.42 & 0.13 & 0.23 & 0.01 \\
\hline$x_{\text {range }}$ & 0.81 & 0.87 & 0.80 & 0.76 & 0.57 & 0.65 & 0.41 & 0.51 \\
\hline$N P L$ & 0.94 & 0.98 & 0.93 & 0.96 & 0.68 & 0.91 & 0.70 & 0.92 \\
\hline NSA & 0.76 & 0.97 & 0.72 & 0.91 & 0.51 & 0.76 & 0.50 & 0.78 \\
\hline$f x_{\text {mean }}$ & 0.59 & 0.80 & 0.57 & 0.89 & 0.28 & 0.62 & 0.21 & 0.63 \\
\hline \multirow[t]{2}{*}{$M-L$} & \multicolumn{2}{|c|}{$v y_{R M S}$} & \multicolumn{2}{|c|}{$a y_{R M S}$} & \multicolumn{2}{|c|}{$v y_{R M S}$} & \multicolumn{2}{|c|}{$a y_{R M S}$} \\
\hline & $E O$ & $E C$ & $E O$ & $E C$ & $E O$ & $E C$ & $E O$ & $E C$ \\
\hline$y_{R M S}$ & 0.94 & 0.963 & 0.79 & 0.91 & 0.94 & 0.52 & 0.90 & 0.37 \\
\hline$y_{\text {range }}$ & 0.97 & 0.969 & 0.85 & 0.91 & 0.98 & 0.67 & 0.89 & 0.50 \\
\hline$N P L$ & 0.88 & 0.993 & 0.81 & 0.94 & 0.97 & 0.93 & 0.93 & 0.86 \\
\hline NSA & 0.91 & 0.971 & 0.74 & 0.93 & 0.92 & 0.84 & 0.91 & 0.70 \\
\hline$f y_{\text {mean }}$ & 0.54 & 0.101 & 0.41 & 0.20 & 0.03 & 0.77 & 0.03 & 0.78 \\
\hline
\end{tabular}

Similarly, in children with cerebral palsy, strong correlations were observed between the $R M S$ of $C o P$ velocity and $C o P$ acceleration and normalized path length and normalized sway area in both eyes open and eyes closed tests. Mild to weak correlations were observed between the $R M S$ of $\mathrm{CoP}$ velocity and $\mathrm{CoP}$ acceleration and $\mathrm{CoP}$ $R M S$, ranges, and mean frequency in the $A-P$ plane. In the $M-L$ plane, all listed metrics showed high correlation with the RMS of $C O P$ velocity and $C o P$ acceleration at the exception of the mean frequency $\left(f y_{\text {mean }}\right)$.

Table 3 shows the Pearson correlation factors between listed new relationships and the mean frequencies $\left(f x_{\text {mean }}, f y_{\text {mean }}\right)$. The $R M S$ of $C o P$ velocity $\left(v x_{R M S}, v y_{R M S}\right)$ and $C o P$ acceleration $\left(a x_{R M S}, a y_{R M S}\right)$ were normalized to the $\operatorname{CoP} R M S$ $\left(x_{R M S}, y_{R M S}\right)$ respectively. The square root of the normalized $C o P$ acceleration $\left(a x_{R M S}, a y_{R M S}\right)$ was strongly correlated to the mean frequencies ( $f x_{\text {mean }}$, $\left.f y_{\text {mean }}\right)$ respectively during eyes open and eyes closed tests in both healthy children and children with cerebral palsy. Similarly, the normalized $R M S$ of $C o P$ velocity $\left(v x_{R M S}, v y_{R M S}\right)$ showed high to strong correlation with the mean frequencies during eyes open and eyes closed test in both 
healthy children and children with cerebral palsy $\left(f x_{\text {mean }}, f y_{\text {mean }}\right)$.

Table 3: Pearson correlation factors between the normalized $R M S$ of $C o P$ velocity and the square root of the normalized $R M S$ of $C o P$ acceleration and the mean frequency respectively in $A-P$ and $M-L$ planes for healthy children and children with cerebral palsy during eyes open and eyes closed tests in quiet standing

\begin{tabular}{l||cc||cc}
\hline & \multicolumn{1}{||}{$\begin{array}{c}\text { Normal } \\
\text { Children } \\
(\boldsymbol{n = 8})\end{array}$} & \multicolumn{2}{|c}{$\begin{array}{c}\text { Children with } \\
\text { CP } \\
(\mathbf{n = 5 4 )}\end{array}$} \\
\hline & $E O$ & $E C$ & $E O$ & $E C$ \\
\hline & 0.971 & 0.978 & 0.846 & 0.860 \\
$f x_{\text {mean }}$ versus $v x_{R M S} / x_{R M S}$ & 0.877 & 0.978 & 0.689 & 0.759 \\
$f x_{\text {mean }}$ versus $\sqrt{a x_{R M S} / x_{R M S}}$ & 0.945 & 0.750 & 0.754 & 0.957 \\
$f y_{\text {mean }}$ versus $v y_{R M S} / y_{R M S}$ & 0.775 & 0.656 & 0.630 & 0.866 \\
$f y_{\text {mean }}$ versus $\sqrt{a y_{R M S} / y_{R M S}}$ & & & & \\
\hline
\end{tabular}

\section{DISCUSSION}

The fast method designed in this study for the computation of sway area has enhanced the ability of processing large amount of $\mathrm{CoP}$ data accurately and quickly without the need for extra computer speed or data output. The correlation analysis between the $C o P$ velocity, acceleration, path length, sway area, and mean frequency have revealed interesting findings. The strong correlation between the $C o P$ velocity and the normalized path length suggested that the CoP velocity metrics may be utilized as an indicator of the normalized path length. The advantage of using the $C o P$ velocity instead of the normalized path length is that the $C o P$ velocity may be measured on line for real time feedback in balance control assessment. Similarly, the strong correlation between the $C o P$ acceleration and the normalized path length and the normalized sway area suggested that the $C o P$ acceleration is equally a valid metric. The normalized sway area is not as strongly correlated to the $\mathrm{CoP}$ velocity and $\mathrm{CoP}$ acceleration.

The weak correlation of $C o P R M S$ and $C o P$ ranges to $C o P$ velocity and $C o P$ acceleration suggested that $R M S$ and ranges values are strongly affected by a slowly varying signal, which is heavily dependent on the initial conditions. The normalization of the $C o P$ velocity and $C o P$ acceleration by the CoP RMS seemed to reduce the effects of the slowly varying signal on the $C o P$ velocity and $C o P$ acceleration and enhance the correlation among the kinematic parameters the mean frequencies. The strong correlation between the normalized $C o P$ velocity and the square root of the normalized $\mathrm{CoP}$ acceleration and the mean frequencies suggested that the effect of the slowly varying signal is further reduced. We concluded that both the normalized $R M S$ of $C o P$ velocity and $\mathrm{CoP}$ acceleration are good indicators of the $\mathrm{CoP}$ energy centroid, and therefore, they can be utilized as complement metrics to $\mathrm{CoP}$ mean frequencies.

\section{CONCLUSION}

The objective of this study is to design a fast method for the computation of sway area and validate the use of $C o P$ velocity and $C o P$ acceleration as fast metrics for online assessment of balance control. The findings of this study suggested that the $C o P$ velocity and $C o P$ acceleration are also important and valid metrics, which can be used in biofeedback and rehabilitation therapy. Furthermore, the $R M S$ of $C o P$ velocity could be utilized as a complement metric to the normalized path length and normalized sway area. The $C o P$ velocity could be utilized as a predictor of the normalized path length and used in real time processing. The normalized $C o P$ velocity and $C o P$ acceleration were also strongly correlated with the mean frequency in both $A-P$ and $M-L$ planes. The normalized $C o P$ velocity and square root of normalized $C o P$ acceleration are good predictors of the mean frequency.

\section{References}

1. Diener W.W.; Dichgans J.; Bacher M.; Gompf B.; Quantification of Postural Sway in Normals and Patients with Cerebellar Diseases, Electroencephalography \& Clinical Neurophysiology, 1984, 57(2), 134-142. 
2. Triolo R.J.; Reilley B.; Freedman W. et al. The Functional Standing Test: Deveolpment and Standardization of a Clinical Evaluation of Standing Function, IEEE Engineering Medicine and Biology, 1992, 11(Dec), 32-34.

3. Triolo R.J.; Bevelheimer T.; Eisenhower G. et al. Development of a Clinical Evaluation of Standing Function, IEEE Transactions on Rehabilitation Engineering, 1993, 1(1), 18-25.

4. Horak F.B. Clinical Assessment of Balance Disorders, Gait \& Posture, 1997, 6(1), 76-84.

5. Burtner P.A.; Qualls C.; Woollacott M.H. Muscle Activation Characteristics of Stance Balance Control in Children with Spastic Cerebral Palsy, Gait \& Posture, 1998, 8(3), 163-174.

6. Liao H.F.; Lai J.S.; Cheng C.K.; Hu M.H. The Relation Between Standing Balance and Walking Function in Children with Spastic Diplegic Cerebral Palsy, Development Medicine \& Child Neurology, 1997, 39, 106112.

7. Hasan S.S.; Robin D.W.; Szurkus D.C.; Ashmead D.H.; Petersen S.W.; Shiavi R.G. Simultaneous Measurement of Body Center of Pressure and Center of Gravity During Upright Stance. Part II: Amplitude and Frequency Data, Gait \& Posture, 1996, 4(1), 11-20.

8. Prieto T.E.; Myklebust L.B.; Myklebust B.M. Characterization and Modeling of Postural Steadiness in the Elderly: A Review, IEEE Transactions on Rehabilitation Engineering, 1993, 1(1), 26-34.

9. Winter D.A.; Patla A.E.; Prince F.; Ishac M.; Gielo-Perczak K. Stiffness Control of Balance in Quiet Standing, Journal of Neurophysiology, 1998, 80(3), 1211-1221.

10. Harris G.F.; Riedel S.A.; Matesi D., Smith P. Standing Postural Stability Assessment and Signal Stationarity in Children with Cerebral Palsy, IEEE Transactions on Rehabilitation Engineering, 1993, 1(1), 35-42.
11. Collins J.J.; DeLuca C.J. Open-loop and Closed-loop Control of Posture: A Randomwalk Analysis of Center-of-pressure Trajectories, Experimental Brain Research, 1993, 95(2), 308-318.

12. Winter D.A.; Prince F., Frank J.S.; Powell C., Zabjek K.F. Unified Theory Regarding A-P and M-L Balance in Quiet Stance, Journal of Neurophysiology, 1996, 75, 2334-2343.

13. Benda B.J.; Riley P.O.; Krebs D.E. Biomechanical Relationship between the Center of Gravity and Center of Pressure During Standing, IEEE Transactions on Rehabilitation Engineering, 1994, 2(1), 3-10.

14. McClenaghan B.A.; Williams H.G.; Dickerson J., Dowda M., Thombs L., Eleazer P. Spectral Characteristics of Aging Postural Control, Gait \& Posture, 1996, 4(3), 112-121.

15. Ferdjallah M.; Harris G.F.; Wertsch J.J. Instantaneous Postural Stability Characterization Using Time-Frequency Analysis, Gait \& Posture, 1999, 10(2), 129134.

16 Oliveira LF, Simpson DM, Nadal J, Calculation of Area of Stabilometric Signals Using Principal Component Analysis, Physiology Measurement, 1996, 17, 305-312.

17 Sevsek F, Determination of Sway Area by Fourier Analysis of itsContour, Proceeding of the $6^{\text {th }}$ WSEAS International Conference on Applied Computer Science, 2006, 514-518.

18 Hassan SS, Robin DW, Shiavi RG, Drugs and Postural Sway, IEEE Engineering in Medicine and Biology, 1992, 11, 35-41.

19 Kiemel T, Oie KS, Jeka JJ, Multisensory Fusion and the Stochastic Structure of Postural Sway, Biological Cybernetics, 2002, 87, 262277.

20 Riach CL, Hayes KC, Maturation of Postural Sway in Young Children, 1987, 29, 650-658. 